\title{
Erratum to: Poplar GATA transcription factor PdGNC is capable of regulating chloroplast ultrastructure, photosynthesis, and vegetative growth in Arabidopsis under varying nitrogen levels
}

Yi An $\cdot$ Xiao Han $\cdot$ Sha Tang $\cdot$ Xinli Xia .

Weilun Yin

Published online: 4 September 2014

(C) Springer Science+Business Media Dordrecht 2014

Erratum to: Plant Cell Tiss Organ Cult

DOI 10.1007/s11240-014-0536-y

In Table 1 of the original publication, the first two column rows for each of the three nitrate levels $(\mathrm{HN}, \mathrm{SN}$ and $\mathrm{LN}$, resp.) were erroneously placed above the headings indicating these levels. The article has been updated to correct this error.

The online version of the original article can be found under doi:10.1007/s11240-014-0536-y.

Y. An $\cdot$ X. Han $\cdot$ S. Tang $\cdot$ X. Xia $(\bowtie) \cdot$ W. Yin

National Engineering Laboratory for Tree Breeding, College of

Biological Sciences and Technology, Beijing Forestry

University, Beijing 100083, China

e-mail: xiaxl@bjfu.edu.cn 